\title{
A combination of STI571 and BCR-ABL1 siRNA with overexpressed p15INK4B induced enhanced proliferation inhibition and apoptosis in chronic myeloid leukemia
}

\author{
D.Y. Xia, L. Liu, M.W. Hao, Q. Liu, R.A. Chen and Y.M. Liang \\ Department of Hematology, Tangdu Hospital, Fourth Military Medical University, Xi'an, China
}

\begin{abstract}
p15INK4B, a cyclin-dependent kinase inhibitor, has been recognized as a tumor suppressor. Loss of or methylation of the p15INK4B gene in chronic myeloid leukemia (CML) cells enhances myeloid progenitor formation from common myeloid progenitors. Therefore, we examined the effects of overexpressed p15INK4B on proliferation and apoptosis of CML cells. Overexpression of p15INK4B inhibited the growth of K562 cells by downregulation of cyclin-dependent kinase 4 (CDK4) and cyclin D1 expression. Overexpression of p15INK4B also induced apoptosis of K562 cells by upregulating Bax expression and downregulating Bcl-2 expression. Overexpression of p15INK4B together with STI571 (imatinib) or BCR-ABL1 small interfering RNA (siRNA) also enhanced growth inhibition and apoptosis induction of K562 cells. The enhanced effect was also mediated by reduction of cyclin D1 and CDK4 and regulation of Bax and $\mathrm{Bcl}-2$. In conclusion, our study may provide new insights into the role of $\mathrm{p} 15 \mathrm{INK} 4 \mathrm{~B}$ in $\mathrm{CML}$ and a potential therapeutic target for overcoming tyrosine kinase inhibitor resistance in $\mathrm{CML}$.
\end{abstract}

Key words: Apoptosis; Chronic myeloid leukemia; p15INK4B; STI571 (Gleevec, imatinib)

\section{Introduction}

Chronic myeloid leukemia (CML) is a myeloproliferative disease originating from a constitutively active tyrosine kinase, BCR-ABL1 (1). The BCR-ABL1 fusion protein is able to disorder the cell regulation system and confer malignant differentiation and proliferation of hematopoietic cells, thus directly contributing to leukemogenesis. Therefore, formation of the BCR-ABL1 fusion gene is a key step in the pathogenesis of $C M L$.

In recent years, inhibition of BCR-ABL1 with tyrosine kinase inhibitors (TKIs) or small interfering RNA (siRNA) has been demonstrated to be an efficient targeted therapy for CML in the chronic phase (2-5). The BCR-ABL1 tyrosine kinase inhibitor, STI571, now called imatinib, is widely used in the treatment of CML $(6,7)$. STI571 may inhibit proliferation and induce apoptosis of CML cells (6). Exposure of K562 cells to morpholino oligo antisense targeted against BCR-ABL1 inhibited proliferation of K562 cells but did not induce apoptosis (3). Zaree Mahmodabady et al. (4) showed that silencing BCR-ABL1 by specific siRNA effectively induced apoptosis of $\mathrm{K} 562$ cells and reduced viability. Although TKIs and targeting of the BCR-ABL1 fusion gene by siRNA have displayed unprecedented efficacy for the treatment of CML (8), there are also many shortcomings that limit the application of these therapeutic methods, such as transfection efficiency, toxicity, and drug resistance $(9,10)$. As a single drug, STI571 has been shown to be ineffective because of drug resistance (11). Although second-generation TKIs such as AMN107 appear to be able to improve the treatment of CML, TKI resistance and relapse also occur frequently in patients (12).

In recent years, combined therapy has become a trend in the treatment of CML. Oh et al. (13) showed that a combination of simvastatin and imatinib exhibited a synergistic killing effect in imatinib-resistant CML cells (13). Ciarcia et al. (14) also reported that phosphatidylinositol 3-kinase (PI3K) and the proto-oncogene SRC kinase inhibitors interacted synergistically with imatinib by inducing apoptosis and autophagy in BCR-ABL1 + leukemia cells. P15INK4B is a cyclin-dependent kinase inhibitor encoded by the CDKN2B gene in humans. P15INK4B forms a complex with CDK4 or CDK6 to prevent the activation of CDKs, and thus functions as a cell growth regulator that inhibits cell 
cycle G1 progression. It has been shown that gene alteration of $\mathrm{p} 15 \mathrm{INK} 4 \mathrm{~B}$ may play an important role in the progress of CML (15). p15INK4B is a downstream gene of the $B C R-A B L 1$ fusion gene (16). BCR-ABL1 may downregulate $\mathrm{p} 15 \mathrm{INK} 4 \mathrm{~B}$ mRNA and protein expression through the PI3K signaling pathway. BCR-ABL1 siRNA and STI571 can not only inhibit BCR-ABL1-induced p15INK4B downregulation and also decrease protein degradation by suppressing the PI3K signaling pathway. Thus, the combination of p15INK4B and BCR-ABL1 inhibitors, TKI or specific siRNA, may be more effective in the treatment of CML. Interestingly, a previous study showed that imatinib, in combination with the p15 gene, displayed an enhanced effect on the inhibition of $\mathrm{K} 562$ cell proliferation and promotion of its apoptosis (17). However, whether BCRABL1 siRNA could enhance the effect of p15INK4B or STI571 on proliferation and apoptosis of K562 cells, and the underlying mechanisms, has not been completely investigated. Therefore, we investigated the effects of $\mathrm{p} 15 \mathrm{INK} 4 \mathrm{~B}$, alone or in combination with BCR-ABL1 inhibitors, on proliferation and apoptosis of K562 cells, and we explored the mechanisms in this study.

\section{Material and Methods}

\section{Construction of plasmid (pcDNA3.1-p15INK4B)}

The CDCBP reference sequence was used for primer designing on Primer Premier 5.0 software, and restriction sites (HindIII and EcoRI) and protective bases were added to the $5^{\prime}$ end of the forward and reverse primers, respectively. The primer sequences were as follows: forward, 5'-GTA AGC TTA TGG CCA CGT CTC TGG ATT TTA-3'; and reverse, 5'-TGG AAT TCT TAA CTA CTA GAC CAA TCT TGA-3'. The estimated length of the product was $465 \mathrm{bp}$. Total RNA was isolated from human peripheral blood mononuclear cells with TRIzol reagent (Invitrogen, USA), and cDNA was synthesized with a firststrand synthesis kit (TaKaRa, China). PCR reaction was performed with the GeneAmp PCR System 9700 (ABI, USA) and started with a polymerase activation step at $94^{\circ} \mathrm{C}$ for $3 \mathrm{~min}$ followed by 30 cycles at $94^{\circ} \mathrm{C}$ for $45 \mathrm{~s}, 55^{\circ} \mathrm{C}$ for $40 \mathrm{~s}$, and $72^{\circ} \mathrm{C}$ for $60 \mathrm{~s}$, and a final extension at $72^{\circ} \mathrm{C}$ for $30 \mathrm{~min}$. The amplified gene with restriction sites was then cloned in mammalian expression vector pcDNA3.1. The constructed pcDNA3.1-p15INK4B plasmid was confirmed through PCR, restriction digestion, and sequencing.

\section{Cell culture and plasmid transfection}

The human leukemia cell line K562 (CML) was obtained from American Type Culture Collection (USA). Cells were maintained in RPMI 1640 supplemented with $10 \%(\mathrm{v} / \mathrm{v})$ heat-inactivated fetal bovine serum and $1 \%$ (v/v) penicillin/streptomycin. K562 cells were incubated at $37^{\circ} \mathrm{C}$ in a humidified atmosphere consisting of $5 \% \mathrm{CO}_{2}$ and $95 \%$ air. All experiments were performed using cells in logarithmic growth phase.
K562 cells stably expressing p15INK4B (K562p15INK4B) were constructed by transfection of pcDNA3.1p15INK4B plasmid with Lipofectamine 2000 (Invitrogen), according to the manufacturer's instructions. After $48 \mathrm{~h}$ of transfection, cells were treated with G418, initially with $500 \mu \mathrm{g} / \mathrm{mL}$ for selecting stable clones then after 14 days with $200 \mu \mathrm{g} / \mathrm{mL}$. The medium was changed every $72 \mathrm{~h}$. Colonies of G418-resistant cells were selected and used in the growth inhibition assay.

\section{Growth inhibition assay}

Growth inhibition of K562 cells was examined using cell-counting kit-8 (Dojindo Laboratories, Japan). Briefly, $1 \times 10^{4}$ cells were seeded on 96 -well plates. After $24 \mathrm{~h}$, the medium was replaced with medium containing STI571 (5 $\mu \mathrm{mol} / \mathrm{L})$. After $24,48,72,96$, and $120 \mathrm{~h}$ of incubation, $10 \mathrm{~mL}$ of cell-counting kit solution was added and incubated for $2 \mathrm{~h}$ in a $\mathrm{CO}_{2}$ incubator. The absorbance of each well was measured with a microplate reader at $450 \mathrm{~nm}$. Means and standard deviation (SD) were generated from three independent experiments. Absorbance values were normalized to the values obtained from the control group to determine the value for percentage survival. Each assay was performed in triplicate.

\section{Apoptosis assay}

Apoptosis of K562 cells was analyzed by flow cytometry using an Annexin V/PI apoptosis kit according to the manufacturer's instructions (Key Technology, Ltd., China). K562 cells were plated onto 6 -well plates $\left(5 \times 10^{5}\right.$ cells/well). The cells, after being treated with STI571 ( $5 \mu \mathrm{mol} / \mathrm{L})$ for $24 \mathrm{~h}$, were washed with phosphate-buffered saline (PBS) 3 times. They were then resuspended in $500 \mu \mathrm{L}$ binding buffer and $5 \mu \mathrm{L}$ Annexin V, and then $2 \mu \mathrm{L}$ $\mathrm{PI}$ was added. Finally, the cells were analyzed using flow cytometry.

\section{Western blot analysis}

Western blot analysis was carried out as described previously (18). Briefly, total protein was isolated with radio-immunoprecipitation assay (RIPA) lysis buffer (Beyotime, China) from K562 cells, and the protein concentration was determined using a bicinchoninic acid (BCA) protein assay kit (Beyotime). Equal amounts of protein were separated by $10 \%$ SDS-PAGE under denaturing and nonreducing conditions and then transferred to a nitrocellulose membrane. The membrane was blocked with $5 \%$ nonfat milk in Tris-buffered saline and Tween 20 (TBST) at room temperature for $1 \mathrm{~h}$ and then incubated with primary antibody at $4^{\circ} \mathrm{C}$ overnight. After they were washed in TBST, the blots were incubated with horseradish-coupled secondary antibody. The signal was detected using enhanced chemiluminescence and recorded on X-ray film. The relative density of target bands was quantified using the Labworks 4.6 image acquisition and analysis software. 


\section{Statistical analysis}

Data are reported as means $\pm S D$. Statistical analysis was performed using ANOVA followed by Fisher's protected least significant differences test, and $\mathrm{P}<0.05$ was considered to be statistically significant.

\section{Results}

Overexpression of p15INK4B alone or in combination with BCR-ABL1 inhibitors inhibited proliferation of K562 cells

As shown in Figure $1 A$ and $B$, overexpression of p15INK4B, BCR-ABL1 siRNA, and STI571 significantly inhibited the proliferation of K562 cells after treatment for indicated times $(P<0.01)$. The cell numbers in p15INK4B combined with BCR-ABL1 siRNA or STI571 groups were less than those in p15INK4B, BCR-ABL1 siRNA, and STI571 groups alone $(P<0.01)$. Similarly, STI571 combined with BCR-ABL1 siRNA also showed an enhanced proliferation inhibition effect on K562 cells than when used alone $(P<0.01$; Figure $1 \mathrm{C})$.

\section{Overexpression of p15INK4B alone or in combination with BCR-ABL1 inhibitors induced K562 cells apoptosis}

Next, we further investigated the effect of overexpression of $\mathrm{p} 15 \mathrm{INK} 4 \mathrm{~B}$ alone or in combination with BCR-ABL1 inhibitors on the apoptosis of K562 cells by flow cytometry. BCR-ABL1 siRNA and STI571 induced an increase in apoptosis of $\mathrm{K} 562$ cells compared with control $(\mathrm{P}<0.01$; Figure 2). Although p15INK4B overexpression increased the apoptosis rate of K562 cells compared with vector, there was no significant difference between the control and vector groups. When the cells with overexpressed p15INK4B were treated with BCR-ABL1 siRNA or STI571, apoptosis rates were further increased compared with BCR-ABL1 siRNA or STI571 alone $(P<0.01)$. When BCR-ABL 1 siRNA was used combined with STI571, the apoptosis rate was also higher than when used alone $(\mathrm{P}<0.01)$.

\section{Effect of overexpression of P15INK4B alone or in combination with BCR-ABL1 inhibitors on cell cycle and apoptosis-related pathways}

To explore the mechanisms underlying the effects of p15INK4B, BCR-ABL1 SiRNA, and STI571 on proliferation and apoptosis of K562 cells, we analyzed apoptosis- and proliferation-related protein expression using Western blot analysis. As shown in Figure 3, p15INK4B, BCR-ABL1 siRNA, and STI571 significantly inhibited expression of cyclin D1 and CDK4. P15INK4B, in combination with BCRABL1 siRNA or STI571, further decreased levels of cyclin D1 and CDK4. P15INK4B, BCR-ABL1 siRNA, and STI571 also significantly upregulated expression of the proapoptotic protein Bax, whereas it downregulated expression of the anti-apoptotic protein Bcl-2. P15INK4B, in combination with BCR-ABL1 siRNA or STI571, also enhanced their effects on expression of Bax and Bcl-2.
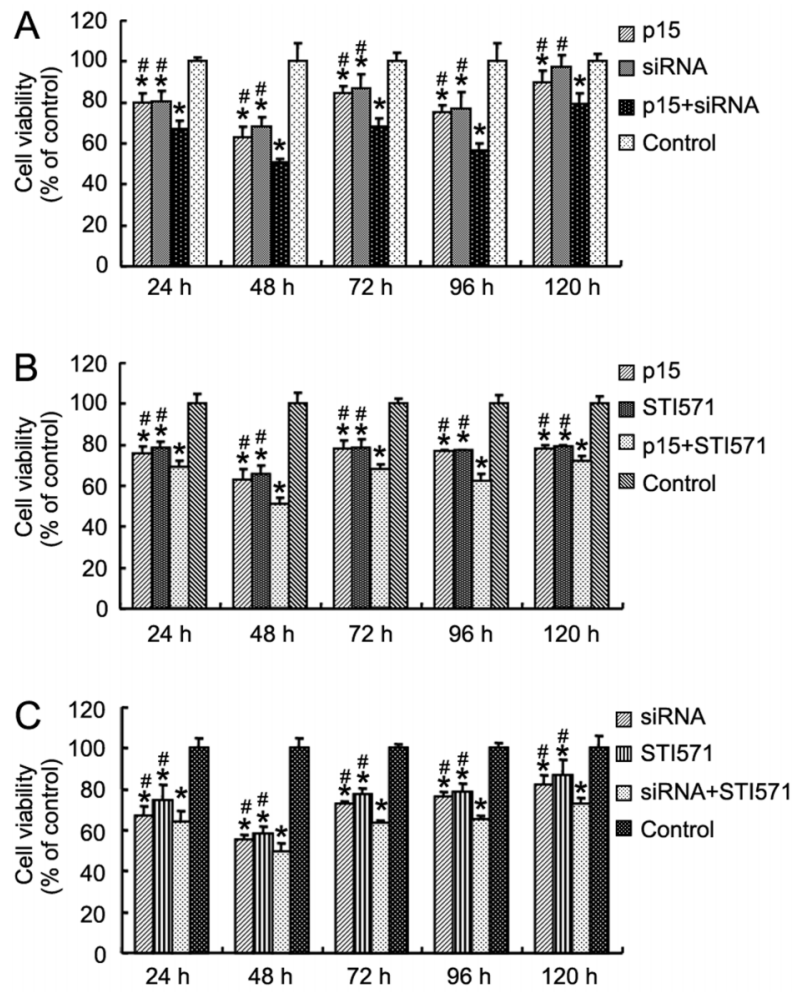

Figure 1. Effect of overexpressed p15INK4B alone or in combination with STI571 and BCR-ABL1 siRNA on the proliferation of K562 cells. A, Effect of $\mathrm{p} 15 \mathrm{INK} 4 \mathrm{~B}$ in combination with BCRABL1 siRNA on the proliferation of K562 cells. BCR-ABL1 siRNA was transiently transfected into K562-p15INK4B cells and K562 cells transfected with pcDNA3.1 (K562/vector) with Lipofectamine 2000. After 24-h incubation, the cells were seeded on 96-well plates with complete RPMI 1640 medium at a density of $1 \times 10^{4}$ cells/well. K562/vector cells transfected with negative siRNA were used as control. MTT assay was performed after incubation for indicated times. $B$, Effect of p15INK4B in combination with STI571 on the proliferation of K562 cells. K562-p15INK4B cells were seeded on 96-well plates with complete RPMI 1640 medium at a density of $1 \times 10^{4}$ cells/well. K562/vector cells were used as control. After treatment with or without STI571 (5 $\mu \mathrm{mol} / \mathrm{L})$ for indicated times, MTT assay was performed. C, Effect of STI571 in combination with BCR-ABL1 siRNA on the proliferation of K562 cells. BCR-ABL1 siRNA was transiently transfected into K562 cells with Lipofectamine 2000. After $24 \mathrm{~h}$, the cells were seeded on 96-well plates with complete RPMI 1640 medium at a density of $1 \times 10^{4}$ cells/well. K562 cells transiently transfected with negative siRNA were used as control. After treatment with or without STI571 $(5 \mu \mathrm{mol} / \mathrm{L})$ for indicated times, MTT assay was performed. Data are reported as means $\pm S D(n=6)$. ${ }^{*} P<0.01$, compared with control; ${ }^{\#} \mathrm{P}<0.01$, compared with combination group (ANOVA followed by Fisher's protected least significant difference test).

\section{Discussion}

Drug resistance is a major obstacle to the successful treatment of CML patients with STI571. Novel agents or drug combinations that overcome this problem are eagerly 

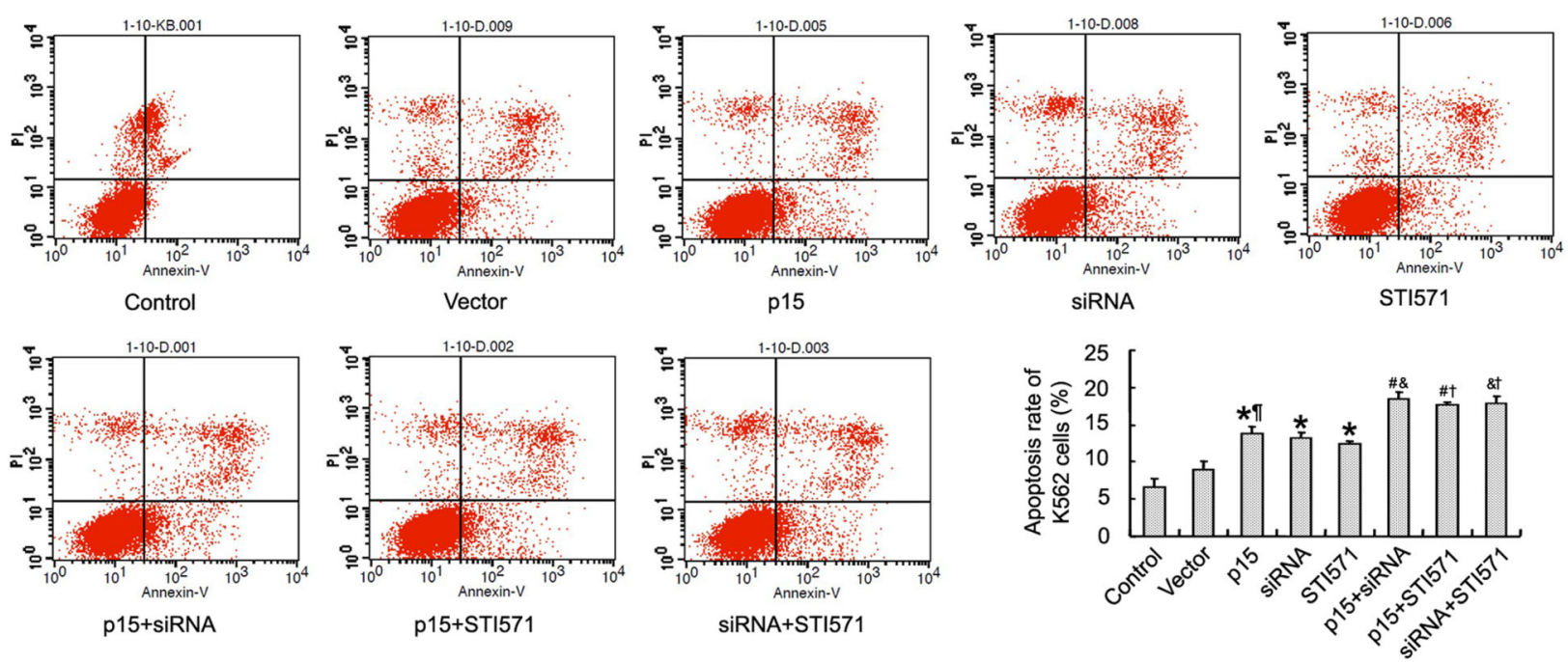

Figure 2. Effect of overexpressed p15INK4B alone or in combination with STI571 and BCR-ABL1 siRNA on the apoptosis of K562 cells. K562 cells were transiently transfected with $\mathrm{p} 15 \mathrm{INK} 4 \mathrm{~B}, \mathrm{BCR}-\mathrm{ABL} 1 \mathrm{siRNA}$ or $\mathrm{p} 15 \mathrm{INK} 4 \mathrm{~B}+\mathrm{BCR}-\mathrm{ABL} 1 \mathrm{siRNA}$. After $24 \mathrm{~h}$, the cells were plated onto 6 -well plates at a density of $1 \times 10^{6}$ cells/well and incubated for another $24 \mathrm{~h}$. Then, the cells were treated with STI571 $(5 \mu \mathrm{mol} / \mathrm{L})$ for $24 \mathrm{~h}$ and flow cytometry analysis was performed to determine the apoptosis of cells. Data are reported as means $\pm \mathrm{SD}$ from 3 independent experiments. ${ }^{*} \mathrm{P}<0.01$, compared with control; " $\mathrm{P}<0.01$, compared with vector group; ${ }^{*} \mathrm{P}<0.01$, compared with p15 group; \& $\mathrm{P}<0.01$, compared with BCR-ABL1 siRNA group; ${ }^{\dagger} \mathrm{P}<0.01$, compared with STI571 group (ANOVA followed by Fisher's protected least significant difference test).

sought. In the present study, our data showed that overexpressed p15INK4B in K562 cells significantly enhanced the effects of proliferation inhibition and apoptosis induction of BCR-ABL1 SiRNA and STI571. In addition, in vitro treatment of $\mathrm{K} 562$ cells with combined BCR-ABL1 SiRNA and STI571 was more effective in inhibiting proliferation and inducing apoptosis than any one used

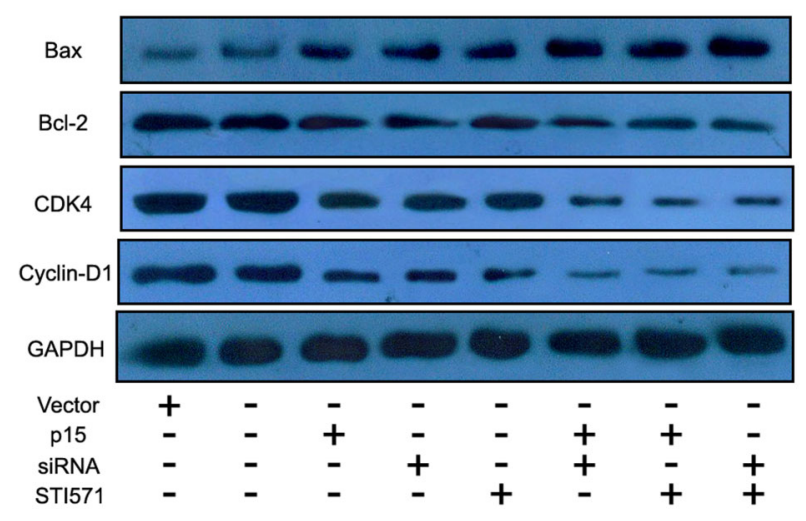

Figure 3. Effect of overexpressed p15INK4B alone or in combination with STI571 and BCR-ABL1 siRNA on cell cycleand apoptosis-related proteins in K562 cells. K562 cells were transiently transfected with $\mathrm{p} 15 \mathrm{INK} 4 \mathrm{~B}, \mathrm{BCR}-\mathrm{ABL} 1$ siRNA or p15INK4B + BCR-ABL1 siRNA. After $24 \mathrm{~h}$, the cells were plated onto 6 -well plates at a density of $1 \times 10^{6}$ cells/well and incubated for another $24 \mathrm{~h}$. Then, the cells were treated with STI571 $(5 \mu \mathrm{mol} / \mathrm{L})$ for $24 \mathrm{~h}$ and Western blot was performed. alone. Western blot analysis showed that overexpressed p15INK4B alone or in combination with STI571 and BCR$A B L 1$ siRNA increased Bax expression and decreased Bcl2 expression in K562 cells. Overexpressed p15INK4B alone or in combination with STI571 and BCR-ABL1 siRNA also inhibited the expression of cell cycle related protein cyclin D1 and CDK4.

Imatinib mesylate has been widely used in the treatment of CML. However, it is becoming clear that a significant portion of patients with long-term imatinib treatment develop drug resistance because of the acquisition of mutations in the kinase domain of BCR-ABL1, which leads to an escape from treatment response (19). p15INK4B is an important inhibitor of CDK4 and CDK6, which play a critical role in the regulation of $\mathrm{G}(1)$-S-phase transition of the cell cycle (20). It has been reported that p15INK4B functions independently as a tumor suppressor for myeloid leukemia development (21). Loss of p15INK4B enhances myeloid progenitor formation from common myeloid progenitors (22). Since the methylation of the p15INK4B promoter is a major gene silencing mechanism in CML (16), restoring the normal expression of p15INK4B should be an effective method in the treatment of CML. Consistent with a previous study (17), our results demonstrated that K562 cells with p15INK4B overexpression showed a lower proliferation rate and higher apoptosis rate than $\mathrm{K} 562 /$ vector cells, indicating that p15INK4B may be a therapeutic target for CML. We also found that overexpression of $\mathrm{p} 15 \mathrm{INK} 4 \mathrm{~B}$ in combination with BCR-ABL1 siRNA or STI571 obviously increased the rate of proliferation inhibition and rate of apoptosis of K562 cells 
compared with when used alone. These results indicate that recovery of $\mathrm{p}$ 15INK4B expression may be a feasible method to overcome TKI resistance in the treatment of CML. Moreover, our study also demonstrated that the combination of BCR-ABL1 siRNA and STI571 displayed a stronger capacity for proliferation inhibition and apoptosis induction of K562 cells than BCR-ABL1 siRNA and STI571 used alone, which suggests that inhibiting both the transcription of the $B C R-A B 1$ fusion gene and the activity of its coded protein may be more effective than BCR-ABL1 siRNA or STI571 used alone.

Cyclin D1/CDK4 promotes G(1)-S-phase transition. Negatively regulating the p15INK4B-cyclin D1/CDK4-RB1mediated pathway is one of the most common and important mechanisms in the growth advantage of tumor cells (23). In the present study, we showed that expression of cyclin D1 and CDK4 in K562 cells with overexpressed p15INK4B was obviously decreased compared with K562 cells transfected with vector, indicating that transfection of p15INK4B reactivates the p15INK4B-cyclin D1/CDK4-RB1 pathway and thereby inhibits proliferation of K562 cells. Besides, STI571 can also inhibit expression of cyclins, such as cyclin D1 and cyclin E. Therefore, we further observed the effect of overexpressed $p 15 I N K 4 B$ in combination with BCR-ABL1 siRNA or STI571 on cyclin D1 and CDK4 expression in K562

\section{References}

1. Leonetti F, Stefanachi A, Nicolotti $O$, Catto M, Pisani L, Cellamare $S$, et al. BCR-ABL inhibitors in chronic myeloid leukemia: process chemistry and biochemical profile. Curr Med Chem 2011; 18: 2943-2959, doi: 10.2174/0929867117 96150414

2. Miller GD, Woessner DW, Sirch MJ, Lim CS. Multidomain targeting of Bcr-Abl by disruption of oligomerization and tyrosine kinase inhibition: toward eradication of CML. Mol Pharm 2013; 10: 3475-3483, doi: 10.1021/mp400323c.

3. Haririan M, Kaviani S, Soleimani M, Ghaemi SR, Delalat B, Atashi A. Morpholino Oligo Antisense efficiently suppresses $B C R / A B L$ and cell proliferation in CML: specific inhibition of BCR-ABL gene expression by Morpholino Oligo Antisense in BCR-ABL $(+)$ cells. Hematology 2012; 17: 28-34, doi: 10.1179/102453311X13127324303470.

4. Zaree Mahmodabady A, Javadi HR, Kamali M, Najafi A, Hojati Z. Bcr-abl silencing by specific small-interference RNA expression vector as a potential treatment for chronic myeloid leukemia. Iran Biomed J 2010; 14: 1-8.

5. Wang Z, Sampath J, Fukuda S, Pelus LM. Disruption of the inhibitor of apoptosis protein survivin sensitizes Bcr-ablpositive cells to STI571-induced apoptosis. Cancer Res 2005; 65: 8224-8232, doi: 10.1158/0008-5472.CAN-05-0303.

6. Niu CC, Zhao C, Zhang XL, Pan J, Zhao C, Wu WR, et al. Wnt5a enhances the response of CML cells to Imatinib Mesylate through JNK activation and gamma-catenin inhibition. Leuk Res 2013; 37: 1532-1537, doi: 10.1016/j.leukres. 2013.07.013.

7. Vander Velde N, Chen L, Guo A, Sharma H, Marynchenko $M, W u E Q$, et al. Study of imatinib treatment patterns and cells. The results showed that the combination of p15INK4B, BCR-ABL1 siRNA, and STI571 exhibited enhanced inhibition of cyclin D1 and CDK4 expression, which may account for the enhanced effect on proliferation of K562 cells. The anti-apoptotic protein $\mathrm{Bcl}-2$ and proapoptotic protein Bax are important apoptosis-associated regulators in the mitochondrial apoptotic pathway (24). Levels of Bcl-2 and Bax may influence the sensitivity of cells to apoptotic stimuli. Thus, the alteration of their expression levels is of great importance in regulating apoptosis (25-27). Our study showed that p15INK4B overexpression, BCR-ABL1 siRNA, and STI571 all increased Bax expression and decreased Bcl-2 expression when used alone. The combination of overexpressed p15INK4B with BCR-ABL1 siRNA and STI571 also enhanced the regulation of $\mathrm{Bax}$ and $\mathrm{Bcl}-2$ expression, resulting in an increase in apoptosis of K562 cells.

In conclusion, our data demonstrated that recovery of p15INK4B expression in CML cells inhibited cell proliferation and induced apoptosis. The combination of p15INK4B overexpression with BCR-ABL1 siRNA and STI571 could enhance cell proliferation and apoptosis due to the regulation of cell cycle- and apoptosis-related proteins. Our study may provide new insight into the role of $\mathrm{p} 15 \mathrm{INK} 4 \mathrm{~B}$ in $\mathrm{CML}$ and a potential therapeutic method to overcome TKI resistance in CML. outcomes among US veteran patients with Philadelphia chromosome-positive chronic myeloid leukemia. J Oncol Pract 2013; 9: e212-e219, doi: 10.1200/JOP.2012.000822.

8. Koldehoff M, Steckel NK, Beelen DW, Elmaagacli AH. Therapeutic application of small interfering RNA directed against bcr-abl transcripts to a patient with imatinib-resistant chronic myeloid leukaemia. Clin Exp Med 2007; 7: 47-55, doi: 10.1007/s10238-007-0125-z.

9. Bengio RM, Riva ME, Moiraghi B, Lanari E, Milone J, Ventriglia $\mathrm{V}$, et al. Clinical outcome of chronic myeloid leukemia imatinib-resistant patients: do BCR-ABL kinase domain mutations affect patient survival? First multicenter Argentinean study. Leuk Lymphoma 2011; 52: 1720-1726, doi: 10.3109/10428194.2011.578310.

10. Swords R, Mahalingam D, Padmanabhan S, Carew J, Giles F. Nilotinib: optimal therapy for patients with chronic myeloid leukemia and resistance or intolerance to imatinib. Drug Des Devel Ther 2009; 3: 89-101.

11. Silva KL, de Souza PS, Nestal de Moraes G, MoellmannCoelho A, Vasconcelos FC, Maia RC. XIAP and Pglycoprotein co-expression is related to imatinib resistance in chronic myeloid leukemia cells. Leuk Res 2013; 37: 13501358, doi: 10.1016/j.leukres.2013.06.014.

12. Xia $\mathrm{Y}$, Fang $\mathrm{H}$, Zhang J, Du $\mathrm{Y}$. Endoplasmic reticulum stress-mediated apoptosis in imatinib-resistant leukemic K562-r cells triggered by AMN107 combined with arsenic trioxide. Exp Biol Med 2013; 238: 932-942, doi: 10.1177/ 1535370213492689.

13. Oh B, Kim TY, Min HJ, Kim M, Kang MS, Huh JY, et al. Synergistic killing effect of imatinib and simvastatin on 
imatinib-resistant chronic myelogenous leukemia cells. Anticancer Drugs 2013; 24: 20-31, doi: 10.1097/CAD. 0b013e32835a0fbd.

14. Ciarcia R, Damiano S, Montagnaro S, Pagnini U, Ruocco A, Caparrotti G, et al. Combined effects of PI3K and SRC kinase inhibitors with imatinib on intracellular calcium levels, autophagy, and apoptosis in CML-PBL cells. Cell Cycle 2013; 12: 2839-2848, doi: 10.4161/cc.25920.

15. Guran S, Bahce M, Beyan C, Korkmaz K, Yalcin A. P53, p15INK4B, p16INK4A and p57KIP2 mutations during the progression of chronic myeloid leukemia. Haematologia 1998; 29: 181-193.

16. Kusy S, Larsen CJ, Roche J. p14ARF, p15INK4b and p16INK4a methylation status in chronic myelogenous leukemia. Leuk Lymphoma 2004; 45: 1989-1994, doi: 10.1080/10428190410001714025.

17. Wang W, Sun BZ, Xie H, Yao LB. [Effect of tyrosine-kinase Inhibitor on p15 gene transfected K562 cells]. Zhongguo Shi Yan Xue Ye Xue Za Zhi 2007; 15: 42-46.

18. Du J, Cheng B, Zhu X, Ling C. Ginsenoside Rg1, a novel glucocorticoid receptor agonist of plant origin, maintains glucocorticoid efficacy with reduced side effects. J Immunol 2011; 187: 942-950, doi: 10.4049/jimmunol.1002579.

19. Gumireddy K, Baker SJ, Cosenza SC, John P, Kang AD, Robell KA, et al. A non-ATP-competitive inhibitor of BCRABL overrides imatinib resistance. Proc Natl Acad Sci U S A 2005; 102: 1992-1997, doi: 10.1073/pnas.0408283102.

20. Ortega S, Malumbres M, Barbacid M. Cyclin D-dependent kinases, INK4 inhibitors and cancer. Biochim Biophys Acta 2002; 1602: 73-87.

21. Bies J, Sramko M, Fares J, Rosu-Myles M, Zhang S, Koller $\mathrm{R}$, et al. Myeloid-specific inactivation of $\mathrm{p} 15$ Ink $4 \mathrm{~b}$ results in monocytosis and predisposition to myeloid leukemia. Blood 2010; 116: 979-987, doi: 10.1182/blood-2009-08-238360.

22. Rosu-Myles M, Taylor BJ, Wolff L. Loss of the tumor suppressor $\mathrm{p} 15 \mathrm{Ink} 4 \mathrm{~b}$ enhances myeloid progenitor formation from common myeloid progenitors. Exp Hematol 2007; 35: 394-406, doi: 10.1016/j.exphem.2006.11.005.

23. Ogino A, Yoshino A, Katayama $Y$, Watanabe $T$, Ota $T$, Komine C, et al. The p15(INK4b)/p16(INK4a)/RB1 pathway is frequently deregulated in human pituitary adenomas. $J$ Neuropathol Exp Neurol 2005; 64: 398-403.

24. Yu J, Zhou X, He X, Dai M, Zhang Q. Curcumin induces apoptosis involving bax/bcl-2 in human hepatoma SMMC7721 cells. Asian Pac J Cancer Prev 2011; 12: 1925-1929.

25. Belka C, Budach W. Anti-apoptotic Bcl-2 proteins: structure, function and relevance for radiation biology. Int J Radiat Biol 2002; 78: 643-658, doi: 10.1080/09553000210137680.

26. Li P, Nijhawan D, Budihardjo I, Srinivasula SM, Ahmad M, Alnemri ES, et al. Cytochrome $\mathrm{c}$ and dATP-dependent formation of Apaf-1/caspase-9 complex initiates an apoptotic protease cascade. Cell 1997; 91: 479-489, doi: 10.1016/ S0092-8674(00)80434-1.

27. Debatin KM, Poncet D, Kroemer G. Chemotherapy: targeting the mitochondrial cell death pathway. Oncogene 2002; 21: $8786-8803$, doi: $10.1038 /$ sj.onc. 1206039. 